\title{
Spherical Symmetric Kink-Like Configurations of Spinor and Gravitational Fields
}

\author{
Jonas Edou $^{1, *}$, Alain Adomou ${ }^{1,2}$, Valerie Ida Senan Hontinfinde ${ }^{1,3}$, Siaka Massou ${ }^{1}$ \\ ${ }^{1}$ Department of Theoretical Physics and Mathematics, University of Abomey-Calavi, Abomey-Calavi, Benin \\ ${ }^{2}$ Higher National Institute of Industrial Technology, University of Abomey, Abomey, Benin \\ ${ }^{3}$ Higher National School of Mathematical Engineering and Modeling, University of Abomey, Abomey, Benin
}

\author{
Email address: \\ edjonas131988@gmail.com (J. Edou) \\ ${ }^{*}$ Corresponding author
}

\section{To cite this article:}

Jonas Edou, Alain Adomou, Valerie Ida Senan Hontinfinde, Siaka Massou. Spherical Symmetric Kink-Like Configurations of Spinor and Gravitational Fields. International Journal of Astrophysics and Space Science. Vol. 8, No. 4, 2020, pp. 32-40.

doi: $10.11648 /$ j.ijass.20200804.11

Received: March 26, 2020; Accepted: August 20, 2020; Published: January 11, 2021

\begin{abstract}
The present research work deals with an extension of a previous work [Exact Soliton-like spherical symmetric solutions of the Heisenberg-Ivanenko type nonlinear spinor field equation in gravitational theory, Journal of Applied Mathematics and Physics, 2020, 8, 1236-1254] to Spherical Symmetric Kink-Like Configurations of Spinor and Gravitational Fields. We have obtained exact kink-like static spherical symmetric solutions to the self-consistent system of spinor and gravitational fields equations. The Einstein's field equations have been solved by the Liouville method. The principal difference between kink soliton with antikink soliton has been established. The nonlinear terms in the lagrangian are arbitrary functions $F\left(I_{S}\right)$ depending on the invariant $I_{S}=S^{2}=(\bar{\psi} \psi)^{2}$. It is shown that the initial set of the Einstein and spinor field equations have regular solutions with a localized energy density of the spinor field only if $m=0$ ( $m$ is the mass parameter in the spinor field equations). Equations with polynomial nonlinearities are thoroughly scrutinized. Let us emphasize that the spinor field with polynomial nonlinearities has a regular solutions with localized, positive and alternating energy density and finite total energy. In addition, the total charge and the total spin are also finte. We have also obtained exact solutions to the linear spinor field equations. We remarked that in this case soliton-like solutions are absent. Furthermore, we note that the properties of regular localized solutions depend on the symmetry and the nonlinear terms in the lagrangian of the self-consistent system of gravitational and spinor fields.
\end{abstract}

Keywords: Lagrangian, Metric, Soliton-Like Solution

\section{Introduction}

The concept of soliton as regular localized stable solutions of nonlinear differential equations is being widely utilized in pure science $[1,2]$. In elementary particle physics, for example, the soliton concept is used as model in order to describe the configuration of elementary particles. The soliton model is dealt with by many autors in general relativity. The solitons with spherical and/or cylindrical symmetry of the interacting system of massless scalar, electromagnetic and gravitational fields are obtained in [3]. An excellent review on the solitons of scalar field with induced nonlinearity and their stability may be found in [4]. For simplicity, the author has considered Friedmann-Robertson-walker and Godel models describing spherically and cylindically symmetric space-time respectively. The plane-symmetric soliton-like solutions are obtained in a series remarkable papers [5-8]. It should be emphasized that, in all these activities, the charge and the spin are not limited. Hence it follows that the own gravitational field of the spinor field is insufficient. The unlimitted problem of the charge and the spin is resolved in a series of interesting article [9-13]. The exact kinklike static plane-symmetric solutions to the self-consistent of interacting scalar, electromagnetic and gravitational fields are obtained in [14]. It was shown that under certain choice of the interaction lagrangian the solutions are regular and have localized energy. The importance of the symmetries in gravitational theory is introduced by Katzin, Lavine and Davis in a series of remarkable articles. The fundamental 
symmetry of the space-time of the general relativity defined by the vanishing Lie derivative of the Riemann curvature tensor is analyzed in [15]. The details literatue on groups of curvature collineation in Riemannian space-times, which admit fields of parallel may found in $[16,17]$. The applications of Lie derivative to symmetries geodesic mappings and first integrals in Riemannian spaces is developped in [18]. The consideration of the proper gravitational field of elementary particles leads to definite the physical interest interpretations of exact solutions obtained because the gravitational field equation is nonlinear by nature. With the introduction of the nonlinear terms in the lagrangian, describing the fields interactions, the field equations possess regular solutions.

The present work, considered as part III of all these investigated initiated in [12], aims and extending the results to spherical symmetric kink-like configurations of spinor and gravitational fields. Here, intead of equations with power nonlinearities examined in [12], we have studied in detail equations with polynomial nonlinearities.

The purpose of the paper was to present some new results in addition to those illustrated in [12] to spherical symmetric kink-like configurations of spinor and gravitational fields.

The paper is organized as follows. Section 2 deals with general equations. The lagrangian of the self-consistent of spinor and gravitational fields and the metric have been defined. From the lagrangian, we obtained the Einstein equations and the spinor field equation. Section 3 addresses the main results. Using the method of Liouville, we solved Einstein equations. In section 4, we discussed the main results by choosing the nonlinear terms in the lagrangian under the polynomial nonlinearities form. Concluding remarks and future work are outlined in section 5.

\section{General Equations}

The lagrangian of the self-consistent system density of the nonlinear spinor and gravitational fields is [6]:

$$
L=\frac{R}{2 \chi}+L_{S p}
$$

where $L_{S p}$ is the spinor field lagrangian. Its expression is defined as follows:

$$
L_{S p}=\frac{i}{2}\left(\bar{\psi} \gamma^{\mu} \nabla_{\mu} \psi-\nabla_{\mu} \bar{\psi} \gamma^{\mu} \psi\right)-m \bar{\psi} \psi+L_{N}
$$

Note that $L_{N}$ is the nonlinear term of $L_{S p}$ which describes the self-interaction of a spinor field. $L_{N}=F\left(I_{S}\right)$ is an arbitrary function depending on the invariant $I_{S}=S^{2}=$ $(\bar{\psi} \psi)^{2} . R=R_{\mu \nu} g_{\mu \nu}$ is the scalar curvature. Then, $\chi=\frac{8 \Pi G}{c^{4}}$ is Einstein's gravitational constant, G is Newton's gravitational constant and $\mathrm{c}$ is the speed of light in vacuum. $\psi$ is the 4components Dirac's spinor with $\bar{\psi}$ its conjugate. The following paragraph will address to the metric.

The metric of space-time admitting spherical symmetric may be written under the following form:

$$
d s^{2}=e^{2 \gamma} d t^{2}-e^{2 \alpha} d \xi^{2}-e^{2 \beta}\left[d \theta^{2}+\sin ^{2}(\theta) d \varphi^{2}\right] .
$$

For simplicity reason, the speed of light has been taken to be unity $(c=1)$. We define spatial variable as in [6] $\xi=\frac{1}{r}$, where $r$ stands for the radial component of the spherical symmetric metric. The metric functions, $\alpha, \beta$ and $\gamma$ are stationnary and are functions of $\xi$ alone. They obey the harmonic coordinate condition as in [6-9]:

$$
\alpha=2 \beta+\gamma
$$

Varying of (1) with respect to the spinor field $\psi$ and its conjugate $\bar{\psi}$ gives nonlinear spinor field equations as follows:

$$
\begin{aligned}
& i \gamma^{\mu} \nabla_{\mu} \psi-m \psi+2 \sqrt{I_{S}} \frac{d F}{d I_{S}} \psi=0, \\
& i \nabla_{\mu} \bar{\psi} \gamma^{\mu}+m \bar{\psi}-2 \sqrt{I_{S}} \frac{d F}{d I_{S}} \bar{\psi}=0,
\end{aligned}
$$

Then, varying of (1) with respect to the metric tensor $g_{\mu \nu}$ leads the general form of Einstein's field equation:

$$
G_{\mu}^{\nu}=R_{\mu}^{\nu}-\frac{1}{2} \delta_{\mu}^{\nu} R=-\chi T_{\mu}^{\nu},
$$

where $G_{\mu}^{\nu}$ is the Einstein's tensor; $R_{\mu}^{\nu}$ is the Ricci's tensor; $\delta_{\mu}^{\nu}$ is the Kronecker's symbol and $T_{\mu}^{\nu}$ is the metric energymomentum tensor of the spinor field. In the sequel, taking into account (1), we obtain the components of the tensor $G_{\mu}^{\nu}$ in the metric (3) under the coordinate condition (4) as in [9]:

$$
\begin{gathered}
G_{0}^{0}=e^{-2 \alpha}\left(2 \beta^{\prime \prime}-2 \gamma^{\prime} \beta^{\prime}-\beta^{2}\right)-e^{-2 \beta}=-\chi T_{0}^{0} \\
G_{1}^{1}=e^{-2 \alpha}\left(2 \beta^{\prime} \gamma^{\prime}+\beta^{\prime 2}\right)-e^{-2 \beta}=-\chi T_{1}^{1} \\
G_{2}^{2}=e^{-2 \alpha}\left(\beta^{\prime \prime}+\gamma^{\prime \prime}-2 \beta^{\prime} \gamma^{\prime}-\beta^{2}\right)=-\chi T_{2}^{2} \\
G_{2}^{2}=G_{3}^{3}, \quad T_{2}^{2}=T_{3}^{3}
\end{gathered}
$$

where prime ( $/$ ) in previous equations means differentiation with respect to $\xi$.

The components of the metric energy-momentum tensor of the spinor field can be written as follows:

$$
T_{\mu}^{\nu}=\frac{i}{4} g^{\nu \rho}\left(\bar{\psi} \gamma_{\mu} \nabla_{\nu} \psi+\bar{\psi} \gamma_{\nu} \nabla_{\mu} \psi-\nabla_{\mu} \bar{\psi} \gamma_{\nu} \psi-\nabla_{\nu} \bar{\psi} \gamma_{\mu} \psi\right)-\delta_{\mu}^{\nu} L_{S} p
$$


Using the spinor field equations (5) and (6), $L_{S p}$ takes the following form:

$$
\begin{aligned}
L_{S_{P}} & =\frac{1}{2} \bar{\psi}\left(i \gamma^{\mu} \nabla_{\mu} \psi-m \psi\right)-\frac{1}{2}\left(i \nabla_{\mu} \bar{\psi} \gamma^{\mu}+m \bar{\psi}\right) \psi+F\left(I_{S}\right) \\
& =-2 S^{2} \frac{\partial F}{\partial I_{S}}+F\left(I_{S}\right) \\
& =-2 I_{S} \frac{\partial F}{\partial I_{S}}+F\left(I_{S}\right) .
\end{aligned}
$$

Taking into account (15), let us write the nontrivial components of the tensor $T_{\mu}^{\nu}$ :

$$
\begin{gathered}
T_{0}^{0}=T_{2}^{2}=T_{3}^{3}=-L_{S p}=2 I_{S} \frac{\partial F\left(I_{S}\right)}{\partial I_{S}}-F\left(I_{S}\right), \\
T_{1}^{1}=\frac{i}{2}\left(\bar{\psi} \gamma^{1} \nabla_{1} \psi-\nabla_{1} \bar{\psi} \gamma^{1} \psi\right)+2 I_{S} \frac{\partial F\left(I_{S}\right)}{\partial I_{S}}-F\left(I_{S}\right) .
\end{gathered}
$$

Let us emphasize that $\gamma^{\mu}$ represent Dirac's matrices in curved space-time. They are linked to Dirac's matrices in flat space-time $\bar{\gamma}_{a}$ by:

$$
\begin{aligned}
g_{\mu \nu}(\xi) & =e_{\mu}^{a}(\xi) e_{\nu}^{b}(\xi) \eta_{a b} \\
\gamma_{\mu}(\xi) & =e_{\mu}^{a}(\xi) \overline{\gamma_{a}}
\end{aligned}
$$

where $\eta_{a b}=\operatorname{diag}(1,-1,-1,-1)$ is the metric of Minkowski and $e_{\mu}^{a}(\xi)$ are tetradic 4-vectors.

With the relation (18), we have:

$$
\gamma^{0}(\xi)=e^{-\gamma} \bar{\gamma}^{0}, \quad \gamma^{1}(\xi)=e^{-\alpha} \bar{\gamma}^{1}, \quad \gamma^{2}(\xi)=e^{-\beta} \bar{\gamma}^{2}, \quad \gamma^{3}(\xi)=\frac{e^{-\beta} \bar{\gamma}^{3}}{\sin \theta}, \quad \gamma^{5}(\xi)=\bar{\gamma}^{5}
$$

The Dirac's matrices in flat space-time are taken in the following way [19, 20]:

$$
\begin{aligned}
& \bar{\gamma}^{0}=\left(\begin{array}{cccc}
1 & 0 & 0 & 0 \\
0 & 1 & 0 & 0 \\
0 & 0 & -1 & 0 \\
0 & 0 & 0 & -1
\end{array}\right) ; \quad \bar{\gamma}^{1}=\left(\begin{array}{cccc}
0 & 0 & 0 & 1 \\
0 & 0 & 1 & 0 \\
0 & -1 & 0 & 0 \\
-1 & 0 & 0 & 0
\end{array}\right) \\
& \bar{\gamma}^{2}=\left(\begin{array}{cccc}
0 & 0 & 0 & -i \\
0 & 0 & i & 0 \\
0 & i & 0 & 0 \\
-i & 0 & 0 & 0
\end{array}\right) ; \quad \bar{\gamma}^{3}=\left(\begin{array}{cccc}
0 & 0 & 1 & 0 \\
0 & 0 & 0 & -1 \\
-1 & 0 & 0 & 0 \\
0 & 1 & 0 & 0
\end{array}\right) \\
& \gamma^{5}=\bar{\gamma}^{5}=\left(\begin{array}{cccc}
0 & 0 & -1 & 0 \\
0 & 0 & 0 & -1 \\
-1 & 0 & 0 & 0 \\
0 & -1 & 0 & 0
\end{array}\right)
\end{aligned}
$$

In the expressions (2), (5)-(6) and (12), $\nabla_{\mu}$ represent the covariant derivative of the spinor meaning. It is connected to the spinor affine connection matrices $\Gamma_{\mu}(\xi)$ as in [21]:

$$
\nabla_{\mu} \psi=\frac{\partial \psi}{\partial \xi^{\mu}}-\Gamma_{\mu} \psi \quad \text { or } \quad \nabla_{\mu} \bar{\psi}=\frac{\partial \bar{\psi}}{\partial \xi^{\mu}}+\Gamma_{\mu} \bar{\psi}
$$

The matrice $\Gamma_{\mu} \psi$, has the following general form:

$$
\Gamma_{\mu}(\xi)=\frac{1}{4} g_{\rho \mu}\left(\partial_{\mu} e_{\sigma}^{b} e_{a}^{\rho}-\Gamma_{\mu \sigma}^{\rho}\right) \gamma^{\delta} \gamma^{\sigma}
$$

In the relation (21), $\Gamma_{\mu \sigma}^{\rho}$ are Christoffel's symbols. According to the expression (21), we have the spinor affine connection matrices:

$$
\Gamma_{0}=-\frac{1}{2} e^{-2 \beta} \bar{\gamma}^{0} \bar{\gamma}^{1} \gamma^{\prime}, \quad \Gamma_{1}=0, \quad \Gamma_{2}=\frac{1}{2} e^{-\beta-\gamma} \bar{\gamma}^{2} \bar{\gamma}^{1} \beta^{\prime}, \quad \Gamma_{3}=\frac{1}{2}\left(e^{-\beta-\gamma} \bar{\gamma}^{3} \bar{\gamma}^{1} \beta^{\prime} \sin \theta+\bar{\gamma}^{3} \bar{\gamma}^{2} \cos \theta\right) .
$$

In virtue of Einstein's convention sommation, we get:

$$
\gamma^{\mu} \Gamma_{\mu}=-\frac{1}{2}\left(e^{-\alpha} \alpha^{\prime} \bar{\gamma}^{1}+\bar{\gamma}^{2} e^{-\beta} \cot \theta\right) .
$$


When we substitute (20) and (23) into (5) (6), we have

$$
\begin{aligned}
& i e^{-\alpha} \bar{\gamma}^{1}\left(\partial_{\xi}+\frac{1}{2} \alpha^{\prime}\right) \psi+\frac{i}{2} \bar{\gamma}^{2} e^{-\beta} \psi \cot \theta-\left(m-2 \sqrt{I_{S}} \frac{d F}{d I_{S}}\right) \psi=0, \\
& i e^{-\alpha} \bar{\gamma}^{1}\left(\partial_{\xi}+\frac{1}{2} \alpha^{\prime}\right) \bar{\psi}+\frac{i}{2} \bar{\gamma}^{2} e^{-\beta} \bar{\psi} \cot \theta+\left(m-2 \sqrt{I_{S}} \frac{d F}{d I_{S}}\right) \bar{\psi}=0 .
\end{aligned}
$$

By choosing the 4-component Dirac spinor under the form $\psi(\xi)=V_{\delta}(\xi)$ with $V_{\delta}(\xi)=\left(\begin{array}{c}V_{1}(\xi) \\ V_{2}(\xi) \\ V_{3}(\xi) \\ V_{4}(\xi)\end{array}\right)$, from (24), we get the following set of equations:

$$
\begin{aligned}
& V_{4}^{\prime}+\frac{1}{2} \alpha^{\prime} V_{4}-\frac{i}{2} e^{\alpha-\beta} V_{4} \cot \theta+i e^{\alpha}\left(m-2 \sqrt{I_{S}} \frac{d F}{d I_{S}}\right) V_{1}=0, \\
& V_{3}^{\prime}+\frac{1}{2} \alpha^{\prime} V_{3}+\frac{i}{2} e^{\alpha-\beta} V_{3} \cot \theta+i e^{\alpha}\left(m-2 \sqrt{I_{S}} \frac{d F}{d I_{S}}\right) V_{2}=0, \\
& V_{2}^{\prime}+\frac{1}{2} \alpha^{\prime} V_{2}-\frac{i}{2} e^{\alpha-\beta} V_{2} \cot \theta-i e^{\alpha}\left(m-2 \sqrt{I_{S}} \frac{d F}{d I_{S}}\right) V_{3}=0, \\
& V_{1}^{\prime}+\frac{1}{2} \alpha^{\prime} V_{1}+\frac{i}{2} e^{\alpha-\beta} V_{1} \cot \theta-i e^{\alpha}\left(m-2 \sqrt{I_{S}} \frac{d F}{d I_{S}}\right) V_{4}=0 .
\end{aligned}
$$

The functions $V_{1}, V_{2}, V_{3}$ and $V_{4}$ are connected by the relation:

$$
V_{1}^{2}-V_{2}^{2}-V_{3}^{2}+V_{4}^{2}=\text { cste } .
$$

\section{Main Results}

Summing the set of equations (26)-(29), we find the firstorder differential equation for the invariant function $I_{S}=S^{2}$ as follows:

$$
\frac{d I_{S}}{d \xi}+2 \alpha^{\prime}(\xi) I_{S}=0 .
$$

The solution of the equation (31) is:

$$
I_{S}(\xi)=C_{0} \exp [-2 \alpha(\xi)], \quad C_{0}=\text { const } .
$$

With the expression (32), we deduce the natural link between the nonlinear spinor field of elementary particles and their own gravitational field.

Using the spinor field equation in the form (24) and the conjugate one, we obtain the following expression for the tensor $T_{1}^{1}$ from the relation (17):

$$
T_{1}^{1}=m \sqrt{I_{S}}-F\left(I_{S}\right)
$$

The following paragraph devotes to the resolution of Einstein's field equations. To this purpose, as the commponents $T_{0}^{0}$ and $T_{2}^{2}$ are equal, we have $G_{0}^{0}-G_{2}^{2}=0$. This leads to the following equation:

$$
\beta^{\prime \prime}-\gamma^{\prime \prime}=e^{2 \beta+2 \gamma},
$$

which can be transformed into a Liouville equation type having the solutions [16]:

$$
\begin{aligned}
\beta(\xi) & =\frac{A}{4}\left(1+\frac{2}{D}\right) \ln \left[\frac{A}{D T^{2}\left(h, \xi+\xi_{1}\right)}\right] \\
& =\left(1+\frac{2}{D}\right) \gamma(\xi), \\
\gamma(\xi) & =\frac{A}{4} \ln \left[\frac{A}{D T^{2}\left(h, \xi+\xi_{1}\right)}\right],
\end{aligned}
$$

$\mathrm{A}$ and $\mathrm{D}$ are integration constants and $\mathrm{T}$ is a function. The function $\mathrm{T}$ has the following form:

$$
T\left(h, \xi+\xi_{1}\right)=\left\{\begin{array}{l}
\frac{1}{h} \sinh \left[h\left(\xi+\xi_{1}\right)\right], h>0 \\
\left(\xi+\xi_{1}\right), h=0 \\
\frac{1}{h} \sin \left[h\left(\xi+\xi_{1}\right)\right], h<0
\end{array}\right.
$$

where $\mathrm{h}$ and $\xi_{1}$ are another unknown integration constants.

By substituting the expressions (35) and (36) into (4), we get the metric function $\alpha(\xi)$ as follows:

$$
\alpha(\xi)=\frac{A}{2}\left(\frac{3}{2}+\frac{2}{D}\right) \ln \left[\frac{A}{D T^{2}\left(h, \xi+\xi_{1}\right)}\right] .
$$

Finally we define the relations between the metric functions $\alpha(\xi), \beta(\xi)$ and $\gamma(\xi)$ :

$$
\beta(\xi)=\frac{2+D}{4+3 D} \alpha(\xi) ; \quad \gamma(\xi)=\frac{D}{4+3 D} \alpha(\xi) .
$$


Equation (9) look likes to the first integral of the equatons (8) and (10). It is also a first order differential equation. Then, introducing (33) and (39)into (9), we have

$$
\left(\alpha^{\prime}\right)^{2}=\frac{(4+3 D)^{2}}{3 D^{2}+8 D+4} e^{2 \alpha}\left[e^{\frac{-4-2 D}{4+2 D} \alpha}-\chi\left(m \sqrt{I_{S}}-F\left(I_{S}\right)\right)\right] .
$$

Taking into account $\alpha^{\prime}=-\frac{1}{2 I_{S}} \frac{d I_{S}}{d \xi}$ and $I_{S}(\xi)=C_{0} e^{-2 \alpha(\xi)}$, from (40) we obtain

$$
\frac{d I_{S}}{d \xi}= \pm \frac{2 \sqrt{C_{0}}(4+3 D)}{\sqrt{3 D^{2}+8 D+4}} \sqrt{I_{S}} \sqrt{\left[\left(\frac{I_{S}}{C_{0}}\right)^{\frac{2+D}{4+3 D}}-\chi\left(m \sqrt{I_{S}}-F\left(I_{S}\right)\right)\right]}
$$

The general solutions of the equation (41) are given by:

$$
\int \frac{d I S}{\sqrt{I_{S}} \sqrt{\left[\left(\frac{I_{S}}{C_{0}}\right)^{\frac{2+D}{4+3 D}}-\chi\left(m \sqrt{I_{S}}-F\left(I_{S}\right)\right)\right]}}= \pm \frac{2 \sqrt{C_{0}}(4+3 D)}{\sqrt{3 D^{2}+8 D+4}}\left(\xi+\xi_{0}\right)
$$

Setting a concrete form of the function $F\left(I_{S}\right)$, from (42) we can determine explicitly $I_{S}(\xi)$. Then, if $I_{S}(\xi)$ is known, we can find the metric function $\alpha(\xi)$ from (32). Finally, we can completely determine the solutions of Einstein equations from the expression (39).

Considering the invariant $I_{S}(\xi)=C_{0} e^{-2 \alpha(\xi)}$, we can establish the regularity properties of the solutions obtained. Studying the distribution of the energy per unit invariant volume $T_{0}^{0} \sqrt{-3_{g}}$, we can establish the localization properties of the solutions.

Let us determine the concrete analytical form of the functions $V_{\delta}(\xi)$. To doing so, we must solve the set of equations (26)-(29) in more compacte form if we pass to the functions $W_{\delta}(\xi)=e^{\frac{\alpha}{2}} V_{\delta}(\xi)$, with $\delta=1,2,3,4$. In this perspective, we obtain:

$$
\begin{aligned}
& W_{4}^{\prime}-\frac{i}{2} e^{\alpha-\beta} W_{4} \cot \theta+i e^{\alpha}\left(m-2 \sqrt{I_{S}} \frac{d F}{d I_{S}}\right) W_{1}=0, \\
& W_{3}^{\prime}+\frac{i}{2} e^{\alpha-\beta} W_{3} \cot \theta+i e^{\alpha}\left(m-2 \sqrt{I_{S}} \frac{d F}{d I_{S}}\right) W_{2}=0, \\
& W_{2}^{\prime}-\frac{i}{2} e^{\alpha-\beta} W_{2} \cot \theta-i e^{\alpha}\left(m-2 \sqrt{I_{S}} \frac{d F}{d I_{S}}\right) W_{3}=0, \\
& W_{1}^{\prime}+\frac{i}{2} e^{\alpha-\beta} W_{1} \cot \theta-i e^{\alpha}\left(m-2 \sqrt{I_{S}} \frac{d F}{d I_{S}}\right) W_{4}=0,
\end{aligned}
$$

where the derivative of the function $W_{\rho}(\xi)$ has the form:

$$
W_{\rho}^{\prime}=\left(V_{\rho}^{\prime}+\frac{1}{2} \alpha^{\prime} V_{\rho}\right) e^{\frac{1}{2} \alpha}
$$

With the set of equations (43)-(46) where $W=W_{\delta}(\xi)$ let us pass to the system of equations depending on functions of the argument $I_{S}$, i.e. $W_{\delta}\left(I_{S}\right)=W_{\delta}(\xi), I_{S}(\xi)=C_{0} e^{-2 \alpha(\xi)}$. We obtain for $W_{\delta}\left(I_{S}\right)$ the set of equations as follows:

$$
\begin{aligned}
& \frac{d W_{4}}{d I_{S}}-i E\left(I_{S}\right) W_{4}+i K\left(I_{S}\right) W_{1}=0 \\
& \frac{d W_{3}}{d I_{S}}+i E\left(I_{S}\right) W_{3}+i K\left(I_{S}\right) W_{2}=0 \\
& \frac{d W_{2}}{d I_{S}}-i E\left(I_{S}\right) W_{2}-i K\left(I_{S}\right) W_{3}=0 \\
& \frac{d W_{1}}{d I_{S}}+i E\left(I_{S}\right) W_{1}-i K\left(I_{S}\right) W_{4}=0
\end{aligned}
$$

where $E\left(I_{S}\right)$ and $K\left(I_{S}\right)$ are defined by the following expressions: 


$$
\begin{aligned}
& E\left(I_{S}\right)=\frac{1}{2} \frac{\left(\sqrt{\frac{C_{0}}{I_{S}}}\right)^{\frac{2+2 D}{4+3 D}} \cot \theta}{\frac{2 \sqrt{C_{0}}(4+3 D)}{\sqrt{3 D^{2}+8 D+4}} \sqrt{I_{S}} \sqrt{\left[\left(\frac{I_{S}}{C_{0}}\right)^{\frac{2+D}{4+3 D}}-\chi\left(m \sqrt{I_{S}}-F\left(I_{S}\right)\right)\right]}} ; \\
& K\left(I_{S}\right)=\frac{\left(\sqrt{\frac{C_{0}}{I_{S}}}\right)\left(m-2 \sqrt{I_{S}} \frac{d F}{d I_{S}}\right)}{\frac{2 \sqrt{C_{0}}(4+3 D)}{\sqrt{3 D^{2}+8 D+4}} \sqrt{I_{S}} \sqrt{\left[\left(\frac{I_{S}}{C_{0}}\right)^{\frac{2+D}{4+3 D}}-\chi\left(m \sqrt{I_{S}}-F\left(I_{S}\right)\right)\right]}}
\end{aligned}
$$

In sequel, we shall transform the equation (48)-(51) to the second order differential equations. In this perspective, differentiating equation (48) and substituting the expression of the function $W_{1}\left(I_{S}\right)$ and the expression of its derivative into the result, we obtain:

$$
W_{4}^{\prime \prime}-\frac{K^{\prime}\left(I_{S}\right)}{K\left(I_{S}\right)} W_{4}^{\prime}+\left[E^{2}\left(I_{S}\right)-K^{2}\left(I_{S}\right)+i \frac{K^{\prime}\left(I_{S}\right) E\left(I_{S}\right)-K\left(I_{S}\right) E^{\prime}\left(I_{S}\right)}{K\left(I_{S}\right)}\right] W_{4}=0 .
$$

Similarly differentiating the equation (51) and introducing into the result the expression of $W_{4}\left(I_{S}\right)$ and the expression of its derivative, we obtain the second-order differential equation for the function $W_{1}\left(I_{S}\right)$ :

$$
W_{1}^{\prime \prime}-\frac{K^{\prime}\left(I_{S}\right)}{K\left(I_{S}\right)} W_{1}^{\prime}+\left[E^{2}\left(I_{S}\right)-K^{2}\left(I_{S}\right)+i \frac{K\left(I_{S}\right) E^{\prime}\left(I_{S}\right)-K^{\prime}\left(I_{S}\right) E^{\prime}\left(I_{S}\right)}{K\left(I_{S}\right)}\right] W_{1}=0 .
$$

Doing the same operating on the equations (49)-(50), we find the second-order differential equations obeyed by the functions $W_{2}\left(I_{S}\right)$ and $W_{3}\left(I_{S}\right)$ as follows:

$$
\begin{aligned}
& W_{3}^{\prime \prime}-\frac{K^{\prime}\left(I_{S}\right)}{K\left(I_{S}\right)} W_{3}^{\prime}+\left[E^{2}\left(I_{S}\right)-K^{2}\left(I_{S}\right)+i \frac{K\left(I_{S}\right) E^{\prime}\left(I_{S}\right)-K^{\prime}\left(I_{S}\right) E^{\prime}\left(I_{S}\right)}{K\left(I_{S}\right)}\right] W_{3}=0 . \\
& W_{2}^{\prime \prime}-\frac{K^{\prime}\left(I_{S}\right)}{K\left(I_{S}\right)} W_{2}^{\prime}+\left[E^{2}\left(I_{S}\right)-K^{2}\left(I_{S}\right)+i \frac{K^{\prime}\left(I_{S}\right) E\left(I_{S}\right)-K\left(I_{S}\right) E^{\prime}\left(I_{S}\right)}{K\left(I_{S}\right)}\right] W_{2}=0 .
\end{aligned}
$$

By summing (54)-(55) and setting $U=W_{1}+W_{4}$, we obtain the following second-order differential equations of the function $U\left(I_{S}\right)$ :

$$
U^{\prime \prime}\left(I_{S}\right)-\frac{K^{\prime}\left(I_{S}\right)}{K\left(I_{S}\right)} U^{\prime}\left(I_{S}\right)+2\left[E^{2}\left(I_{P}\right)-K^{2}\left(I_{S}\right)\right] U\left(I_{S}\right)=0 .
$$

The equation (58) may be transformed to:

$$
\frac{1}{K\left(I_{S}\right) \sqrt{2 \varepsilon}} \frac{d}{d I_{S}}\left[\frac{U^{\prime}\left(I_{S}\right)}{K\left(I_{S}\right) \sqrt{2 \varepsilon}}\right]-U\left(I_{S}\right)=0
$$

under the condition $E^{2}\left(I_{S}\right)=(1-\varepsilon) K^{2}\left(I_{S}\right)$ with $0<\varepsilon \leq 1$.

The first integral of the equation (59) is

$$
U^{\prime}\left(I_{S}\right)= \pm \sqrt{U^{2}\left(I_{S}\right)+C_{1}} K\left(I_{S}\right) \sqrt{2 \varepsilon}, \quad C_{1}=\text { const. }
$$

If $C_{1}=a_{1}^{2}>0$, then the equation (60) has the solution

$$
U\left(I_{S}\right)=a_{1} \sinh N_{1}\left(I_{S}\right) .
$$

If $C_{1}=-b_{1}^{2}<0$, the solution of the equation (58) is given by:

$$
U\left(I_{S}\right)=b_{1} \cosh N_{1}\left(I_{S}\right) .
$$

with

$$
N_{1}\left(I_{S}\right)=\sqrt{2 \varepsilon} \int K\left(I_{S}\right) d I_{S}+R_{1}, \quad R_{1}=\text { const } .
$$


The difference of equations (48) and (51), taking into account of (61) and (62), gives:

$$
X\left(I_{S}\right)=W_{1}-W_{4}=-i a_{1}\left(\frac{\sqrt{1-\varepsilon}-1}{\sqrt{2 \varepsilon}}\right) \cosh N_{1}\left(I_{S}\right),
$$

or

$$
X\left(I_{S}\right)=W_{1}-W_{4}=-i b_{1}\left(\frac{\sqrt{1-\varepsilon}-1}{\sqrt{2 \varepsilon}}\right) \sinh N_{1}\left(I_{S}\right)
$$

where $a_{1}$ and $b_{1}$ are integration constants.

Solving analogously the equations (56) and (57), we obtain the following expressions for $Y\left(I_{S}\right)=W_{2}+W_{3}$ as follows:

$$
Y\left(I_{S}\right)=a_{2} \sinh N_{2}\left(I_{S}\right), \quad \text { for } \quad C_{2}=a_{2}^{2}>0
$$

or

$$
Y\left(I_{S}\right)=b_{2} \cosh N_{2}\left(I_{S}\right), \quad \text { for } \quad C_{2}=-b_{2}^{2}<0 .
$$

In these conditions, it then follows from the expressions (66) and (67) that:

$$
V\left(I_{S}\right)=W_{2}-W_{3}=i a_{2}\left(\frac{\sqrt{1-\varepsilon}-1}{\sqrt{2 \varepsilon}}\right) \cosh N_{2}\left(I_{S}\right),
$$

or

$$
\begin{gathered}
V\left(I_{S}\right)=W_{2}-W_{3}=i b_{2}\left(\frac{\sqrt{1-\varepsilon}-1}{\sqrt{2 \varepsilon}}\right) \sinh N_{2}\left(I_{S}\right), \\
N_{2}\left(I_{P}\right)=\sqrt{2 \varepsilon} \int H\left(I_{P}\right) d I_{P}+R_{2},
\end{gathered}
$$

where $a_{2}, b_{2}$ and $R_{2}$ are integration constants.

Considering the cases where $C_{1}=a_{1}^{2}>0$ and $C_{2}=-b_{2}^{2}<0$, let us determine the expressions of the functions $W_{\delta}\left(I_{S}\right)$. We get for the functions $W_{\delta}\left(I_{S}\right)$ the following expressions:

$$
\begin{aligned}
& W_{1}\left(I_{S}\right)=a_{0}\left[\sinh N_{1}\left(I_{S}\right)-i\left(\frac{\sqrt{1-\varepsilon}-1}{\sqrt{2 \varepsilon}}\right) \cosh N_{1}\left(I_{S}\right)\right], \\
& W_{2}\left(I_{S}\right)=b_{0}\left[\cosh N_{2}\left(I_{S}\right)+i\left(\frac{\sqrt{1-\varepsilon}-1}{\sqrt{2 \varepsilon}}\right) \sinh N_{2}\left(I_{S}\right)\right], \\
& W_{3}\left(I_{S}\right)=b_{0}\left[\cosh N_{2}\left(I_{S}\right)-i\left(\frac{\sqrt{1-\varepsilon}-1}{\sqrt{2 \varepsilon}}\right) \sinh N_{2}\left(I_{S}\right)\right], \\
& W_{4}\left(I_{S}\right)=a_{0}\left[\sinh N_{1}\left(I_{S}\right)+i\left(\frac{\sqrt{1-\varepsilon}-1}{\sqrt{2 \varepsilon}}\right) \cosh N_{1}\left(I_{S}\right)\right],
\end{aligned}
$$

with $a_{0}=\frac{1}{2} a_{1}$ and $b_{0}=\frac{1}{2} b_{2}$.

Let us note that we can also obtain the expressins of the funstions $W_{\delta}\left(I_{S}\right)$ considering $C_{1}=-b_{1}^{2}<0$ and $C_{2}=a_{2}^{2}>0$. Furthermore, in the relations (63) and (70), without loss of generality we can use the minus sign before the integral. Let us pass to the functions $V_{\delta}(\xi)$ by multiplying the functions $W_{\delta}(\xi)$ obtained in the expressions (71)-(74) by $e^{-\frac{1}{2} \alpha(\xi)}$ as follows:

$$
\begin{aligned}
& V_{1}(\xi)=a_{0}\left[\sinh N_{1}(\xi)-i\left(\frac{\sqrt{1-\varepsilon}-1}{\sqrt{2 \varepsilon}}\right) \cosh N_{1}(\xi)\right] \exp \left\{-\frac{A}{4}\left(\frac{3}{2}+\frac{2}{D}\right) \ln \left[\frac{A}{D T^{2}\left(h, \xi+\xi_{1}\right)}\right]\right\} \\
& V_{2}(\xi)=b_{0}\left[\cosh N_{2}(\xi)+i\left(\frac{\sqrt{1-\varepsilon}-1}{\sqrt{2 \varepsilon}}\right) \sinh N_{2}(\xi)\right] \exp \left\{-\frac{A}{4}\left(\frac{3}{2}+\frac{2}{D}\right) \ln \left[\frac{A}{D T^{2}\left(h, \xi+\xi_{1}\right)}\right]\right\} \\
& V_{3}(\xi)=b_{0}\left[\cosh N_{2}(\xi)-i\left(\frac{\sqrt{1-\varepsilon}-1}{\sqrt{2 \varepsilon}}\right) \sinh N_{2}(\xi)\right] \exp \left\{-\frac{A}{4}\left(\frac{3}{2}+\frac{2}{D}\right) \ln \left[\frac{A}{D T^{2}\left(h, \xi+\xi_{1}\right)}\right]\right\} \\
& V_{4}(\xi)=a_{0}\left[\sinh N_{1}(\xi)+i\left(\frac{\sqrt{1-\varepsilon}-1}{\sqrt{2 \varepsilon}}\right) \cosh N_{1}(\xi)\right] \exp \left\{-\frac{A}{4}\left(\frac{3}{2}+\frac{2}{D}\right) \ln \left[\frac{A}{D T^{2}\left(h, \xi+\xi_{1}\right)}\right]\right\}
\end{aligned}
$$


Using analytical expressions of the functions $V_{\delta}(\xi)$, the total charge $Q$ and spin $S_{1}$ have been determined in [12]. It has been emphasized that the total charge and spin are limited.

In the following section, we shall deal with the analysis of the main results by choosing the concrete form of the nonlinear terms $F\left(I_{S}\right)$.

\section{Discussion}

The equations with power nonlinearities have been thoroughly discussed by us in [12] where we have obtained exact soliton-like spherical symmetric solutions of the Heisenberg-Ivanenko type nonliear spinor field equation in gravitational theory. In this present analysis, the nonlinear terms $F\left(I_{S}\right)$ in the lagrangian have been chosen under the polynomial nonlinearities form as follows:

$$
F\left(I_{S}\right)=\lambda\left(\sqrt{\frac{I_{S}}{\omega_{0}^{2}}}-1\right)^{2}\left(2-\sqrt{\frac{I_{S}}{\omega_{0}^{2}}}\right)^{2} .
$$

where $\lambda$ is a nonlinearity parameter.

In this optic, the nonlinear terms $F\left(I_{S}\right)$ admit two nonzero roots $I_{S}=\omega_{0}^{2}$ and $I_{S}=4 \omega_{0}^{2}$.

Without losing the generality, we have chosen the massless elementary particles $(m=0)$ [22] and we have assumed that $\frac{1}{C_{0}} \longrightarrow 0$.

Under the assumption made above, by substituting (79) into (42), we have obtained the analytical expression of the invariant function $I_{S}$ :

$$
\begin{gathered}
I_{S_{1}}=\omega_{0}^{2}\left[2-\frac{1}{\exp \left[\zeta_{0}\left(\xi+\xi_{0}\right)\right]}\right]^{2}, \\
I_{S_{2}}=\omega_{0}^{2}\left[2-\frac{1}{\exp \left[-\zeta_{0}\left(\xi+\xi_{0}\right)\right]}\right]^{2},
\end{gathered}
$$

where

$$
\zeta_{0}=\frac{C_{0} \lambda(4+3 D)}{\omega_{0} \sqrt{3 D^{2}+8 D+4}}=\text { const. }
$$

From the relations (80) and (81), the function $I_{v}$ is regular. Therefore, as $e^{2 \alpha}=\frac{C_{0}}{I_{S}}$, according to (31), the metric is regular everywhere for $\xi \in\left[0, \xi_{C}\right]$. The energy density is given by the following expression:

$$
T_{0(1,2)}^{0}=\frac{\lambda e^{ \pm \zeta_{0}\left(\xi+\xi_{0}\right)}\left[2+e^{ \pm \zeta_{0}\left(\xi+\xi_{0}\right)}-4 e^{ \pm 2 \zeta_{0}\left(\xi+\xi_{0}\right)}\right]}{\left[1+e^{ \pm \zeta_{0}\left(\xi+\xi_{0}\right)}\right]^{4}}
$$

From the expression (82), the energy density $T_{0}^{0}(\xi)$ of nonlinear spin field is a positive, alternating, regular and localized function. Moreover, the total energy $E=$ $\int_{0}^{\xi_{c}} T_{0(1,2)}^{0} \sqrt{3_{-g}} d \xi$ is positive and finite.

We note that the functions $I_{S_{1}}$ and $I_{S_{2}}$ describe the kink and antikink solitons-like solutions. The indices 1 and 2 refer to kink and antikink in the expression of the energy density $T_{0}^{0}(\xi)$. The study of the kink and antikink configurations in plane-symmetric metric may found in [6].

\section{Concluding Remarks}

Taking into account the proper gravitational field of elementary particles, we obtained the spherical symmetric kink-like solutions of nonlinear spinor and Einstein equations when the nonlinear terms in the larangian is chosen under the polynomial nonlinearities form. These solutions describe a nonlinear spinor field configuration with localized energy density $T_{0}^{0}$, positive energy $E$ and a regular metric $d s^{2}$. The forthcoming paper will deal with Spherical symmetric solitons of interacting spinor and scalar fields in general relativity theory.

\section{Conflicts of Interest}

All the authors do not have any possible conflicts of interest.

\section{References}

[1] Scott, A. C., Chu, F. Y. F., and McLaughlin, D. W. (1973). Proc. IEEE61, 1443.

[2] Shikin, G. N. (1995) Theory of Solitons in General Relativity. URSS, Moscow.

[3] B. Saha, gr-qc/9811044v1, (1998).

[4] Saha (2000) Soliton of scalar field with induced nonlinearity and their stability. International Journal of Modern Physics A, 15, No. 10, 1481-1496.

[5] Adomou, A., Alvarado, R. and Shikin, G. N. (1995), Nonlinear spinor field equations in gravitaional theory, Izvestiya Vuzov. Fizika, 863-868.

[6] Adomou, A. and Shikin, G. N. (1998), Exact static plane symmetric solutions to the nonlinear spinor field equations in the gravitational theory, Izvestia VUZov, Fizika, 41, 69.

[7] saha, B. and Shikin, G. N. (2003), plane symmetric solitons of spinor and scalar fields, Czechoslovak Journal of Physics, 54, 597-620. https://doi.org/10.1023/B:CJOP.0000029690.61308.a5

[8] Adomou, A., Edou, J. and Massou, S. (2019), plane symmetric solutions to the nonlinear spinor field equations in general relativity theory Journal of Modern Physics, 10, 1222-1234. https://doi.org/10.4236/jmp.2019.1010081

[9] Adanhoumè, A., Adomou, A., Codo, F. P. and Hounkonnou, M. N. (2012), Nonlinear spinor field equations in gravitaional theory: spherical symmetric soliton-like solutions, Journal of Modern Physics, 3, 935. https://doi.org/10.4236/jmp.2012.39122 
[10] Adomou, A., Edou, J. and Massou, S. (2019), Solitonlike spherical symmetric solutions of the nonlinear spinor field equations depending on the invariant $I_{P}$ in the general relativity Theory, Journal of Applied Mathematics and Physics, 7, 2818-2835.

[11] Massou, S., Adomou, A. and Edou, J. (2019), Soliton-like spherical symmetric solutions of the nonlinear spinor field equations in general relativity International Journal of Applied Mathematics and Theoretical Physics. Vol.5, N0.4,2019,pp.118-128. doi:10.11648/j.ijamtp.20190504.14.

[12] Adomou, A., Edou, J., Hontinfinde S. I. V. and Massou, S. (2020), Exact Soliton-Like Spherical Symmetric solutions of Heisenberg-Ivanenko Nonlinear Spinor Field Equation in Gravitational Theory, Journal of Applied Mathematics and Physics, 8, 1236-1254.

[13] Adomou, A., Edou, J., Hontinfinde S. I. V. and Massou, S. (2020), SPHERICAL SYMMETRIC SOLITONS OF SPINOR FIELD IN GRAVITATIONAL THEORY, International Journal of Advanced Research, 8 (6), 1331 1340 .

[14] Kulyabov D.S., Rybakov Yu. P., Shikin G. N., Yuschenko L.P., math-ph/9902011v1, (1999).

[15] Katzin, G. H., Livine, J. and Davis, W. R. (1969) Curvature collineation: A fundamental symmetry property of the space-times of the general relativity defined by the vanishing Lie derivative of the Riemann curvature tensor. Journal of Mathematical Physics, 10, 617-620.

[16] Katzin, G. H., Livine, J. and Davis, W. R. (1970) Groups of Curvature collineation in Riemannian spacetimes which admit fields of parallel vectors. Journal of Mathematical Physics, 11, 1578-1580.

[17] Katzin, G. H.an dLivine, J. Applications of Lie derivatives to symmetries, geodesic mappings, and first integrals in Riemannian spaces. Collection of articles commemorating Wladyslaw Slebodzinski. Colloquium Mathematicum XXVI 26 (1972) 21, Tensor (N. S.), 22 (1971) 64.

[18] Norris, L. K., Green, L. H. and Davis, W. R. Fluid space-times including electromagnetic fields admitting symmetry mappings belonging to the family of contracted Ricci collineations.Journal of Mathematical Physics. 18 (1977), no.7, 13051311.

[19] Zhelnorovich, V. A. (1982) Theory of spinors and its applications to Physics and Mechanics. Nauka, Moscow.

[20] Bogoliubov, N. N. and Shirkov, D. V. (1976) Introduction to the theory of Quantized Fields. Nauka, Moscow.

[21] D. Brill and J.Wheeler, Rev.Mod.Phys. 29, 465 (1957).

[22] Heisenberg, W. , (1966) Introduction to Unified Field Theory of Elementary Particles. Interscience Publishers, London. 